REVIEW ARTICLE

S. Geibprasert

M. Gallucci

T. Krings

\section{Addictive Illegal Drugs: Structural Neuroimaging}

\begin{abstract}
SUMmARY: Illegal addictive drugs can lead to functional or structural impairment of the central nervous system. This review provides an overview of the structural imaging findings on CT, MR imaging, and conventional angiography related to chronic and acute abuse of the most commonly abused illegal drugs, including cannabis, organic solvents, and amphetamines and opioids and their respective derivatives. Pathomechanisms include excitotoxicity, which may lead to an acute or subacute leukoencephalopathy, and vascular complications, including vasoconstriction, vasculitis, or hypertension, which may lead to intracranial hemorrhage or ischemia. Because clinical findings alone are often nonspecific, and afflicted patients are unlikely to admit to the substance abuse, the neuroradiologist may play an important role in establishing the diagnosis and, thereby, initiating treatment.
\end{abstract}

ABBREVIATIONS: $\mathrm{CB}=$ cannabinoid; 5 -HT = serotonin (5-hydroxytryptamine); $\mathrm{MCA}=$ middle cerebral artery; MDMA = 3,4-methylenedioxymethamphetamine; MRA = MR angiography; $\mathrm{PET}=$ positron-emission tomography lis llegal addictive drugs can alter both the function and the structure of the central nervous system. ${ }^{1-3}$ Functional imaging studies, such as MR spectroscopy, functional MR imaging, single-photon emission tomography, or PET may detect changes in the regional cerebral blood flow, cerebral activity, and neuropharmacologic parameters, such as receptor number and neurotransmitter levels. ${ }^{4,5}$ These methods are, therefore, of importance in detecting the functional or metabolic impairment following illegal drug abuse. On the other hand, conventional imaging methods (ie, CT, MR imaging, or angiography) will demonstrate the structural damage done to the brain. These structural alterations may be due to acute or chronic toxicity, they may be reversible or irreversible, and their pattern of involvement may be specific or nonspecific for the substance used. Because clinical symptoms are often nonspecific and may mimic other etiologies and because the correct diagnosis is of great importance to prevent further damage to the brain, the neuroradiologist plays a crucial role in raising the suspicion of illegal drug abuse. This review describes the most commonly encountered addictive drug-induced changes to the brain, their pathomechanisms, clinical findings, and, most important, their imaging features, focusing on structural rather than functional imaging studies.

\section{Cannabis}

Cannabis or marijuana is the most commonly used illicit drug worldwide. ${ }^{6,7}$ The major psychoactive compound is $\delta$-9-tetrahydrocannabinol, which is a lipid-soluble substance that causes activation of the $\mathrm{CB}$ receptors. The $\mathrm{CB}$ receptors are heterogeneously distributed in the brain, with the highest densities in the basal ganglia, substantia nigra, globus pallidus, dentate gyrus of

From the Division of Neuroradiology (S.G.), Department of Diagnostic Radiology, University of Toronto, Hospital for Sick Children, Toronto, Ontario, Canada; Department of Neuroradiology (M.G.), University Hospital "S. Salvatore," L'Aquila, Italy; and Division of Neuroradiology (T.K.), Department of Medical Imaging, University of Toronto, Toronto Western Hospital, Toronto, Ontario, Canada.

Please address correspondence to T. Krings, MD, PhD, University of Toronto, Toronto Western Hospital, UHN, Division of Neuroradiology, 399 Bathurst St, 3MCL-429, Toronto, ON, M5T 2S8, Canada; e-mail: timo.krings@uhn.on.ca

Indicates open access to non-subscribers at www.ajnr.org

DOI 10.3174/ajnr.A1811 hippocampus, limbic cortices, and cerebellum. ${ }^{8}$ Smoking is the preferred route of drug delivery, but it can also be taken orally.

Acute effects of cannabis include cognitive dysfunction, memory and time-assessment alterations, motor incoordination, poor executive functions, and sedative effects. ${ }^{9}$ Cardiovascular changes occur for about 2-3 hours following drug abuse, with an increased heart rate and cardiac output, increased blood pressure in the supine position, and orthostatic hypotension. Chronic use can lead to behavioral changes and seems to increase the risk for developing schizophrenia.

Ischemic strokes related to cannabis have been reported in the literature; however, due to the widespread use of the drug and confounding problems of polydrug abuse, it is difficult and often impossible to establish whether these strokes are truly associated with cannabis or other ingested drugs or are purely coincidental. Vasospasm, orthostatic hypotension, and vasculitis have been proposed as mechanisms in young patients without other vascular risk factors. ${ }^{10}$ Functional imaging studies, including PET and MR perfusion, in the acute stage of cannabis abuse demonstrate an overall decrease in regional cerebral blood flow in the frontal, parietal, temporal, and occipital lobes, ${ }^{11,12}$ while transcranial Doppler shows an increased pulsatility index due to increased resistance from vasoconstriction. ${ }^{13}$ The stroke pattern reported following cannabis ingestion is nonspecific, and imaging findings are indistinguishable from those in strokes of other causes.

\section{Organic Solvents: Inhalants (Toluene)}

Toluene or methyl-benzene is a lipid-soluble aromatic hydrocarbon, which is commonly used as a solvent in many industrial products such as paint, paint thinner, glue, or ink. It is widely abused in many countries ${ }^{14,15}$ due to its widespread availability and low cost. There are hardly any discernible withdrawal symptoms. Long-term occupational exposures, for example, in dry cleaning, aviation, and chemical industries, may lead to chronic intoxication. The lungs rapidly absorb the inhaled toluene into the blood, and the substance subsequently accumulates in the lipid-rich brain. ${ }^{16}$ Toluene is oxidized into benzolic acid before being conjugated with glycine in the liver to form hippuric acid, which is excreted by the kidneys.

Acute intoxication following inhalation is clinically characterized by reversible behavioral changes, euphoria, headache, and ataxia (afflicted subjects are referred to as "spray 


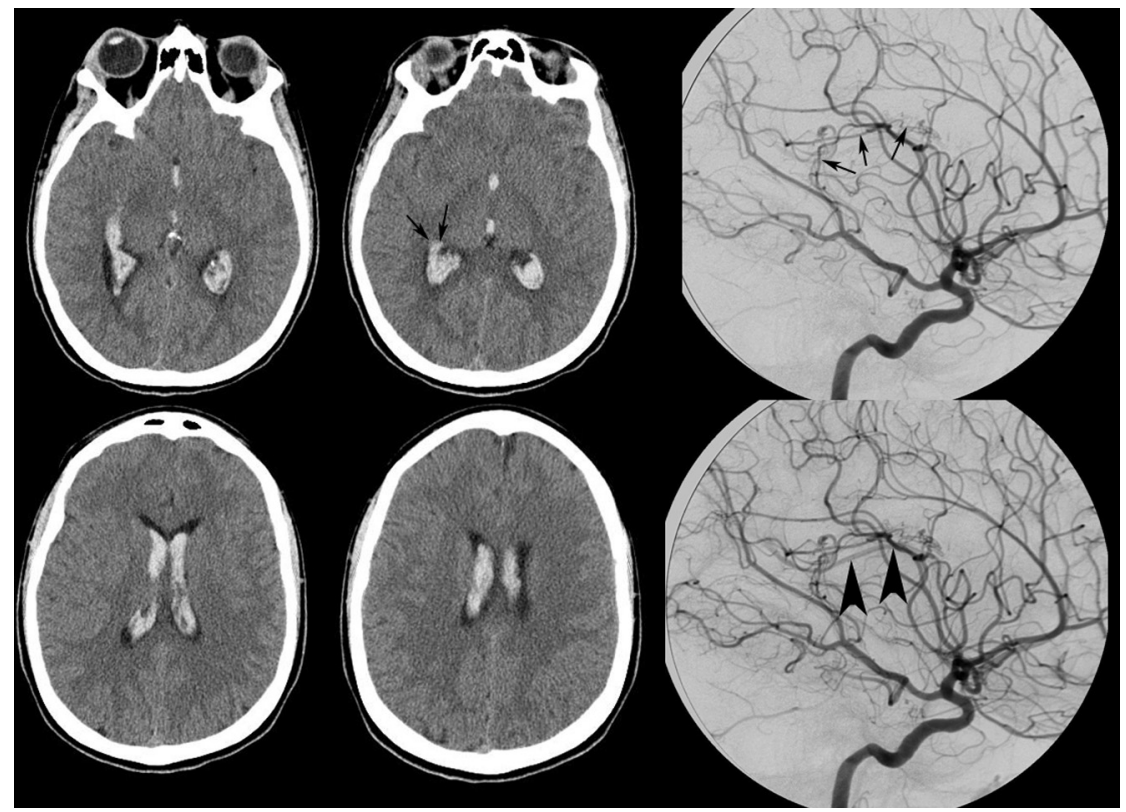

Fig 1. A 19-year-old woman presented with acute onset of headaches to the emergency department. CT demonstrates an intraventricular hemorrhage with a potential parenchymal component close to the right ventricular wall (arrows on CT) Drug screening was positive for alcohol and cocaine. Because approximately half of the patients with intracrania hemorrhage following cocaine abuse have an underlying cause for their hemorrhage, angiography was performed demonstrating a choroidal arteriovenous malformation fed by the posterior lateral choroidal artery (arrows on angiography) of the posterior cerebral artery, with slightly early venous filling (arrowheads), which was subsequently successfully embolized.

heads"). In the acute stage, no alterations are seen on structural imaging; in chronic abusers, however, irreversible neurologic damage with structural alterations will occur. ${ }^{17,18}$ Findings of toluene-induced chronic toxic encephalopathy include changes in cerebellar and cerebral white matter, with demyelination and gliosis, which leads to cerebellar dysfunction; psychiatric disorders; spasticity; cognitive changes; and secondary Parkinson disease. Symptoms of attention deficit; decline in visual-spatial skills, frontal lobe function, and memory retrieval; hearing and visual loss; and cranial nerve abnormalities may also occur. Long-term exposure will result in brain atrophy, predominantly in the cerebellum, owing to a decline of Purkinje cells in the brain stem at the pontine level and in the hippocampus. There are few reports of optic nerve atrophy.

Abnormal findings on MR imaging are typically seen after at least $4-7$ years of chronic inhalant abuse and are irreversible once detected, suggesting that the lesions are related to the accumulative toxic effect of the substance. Diffuse T2 hyperintensities can be seen in the periventricular white matter, centrum semiovale and the cerebellum, internal capsule, and pons. These white matter lesions are concordant with the severity of neurologic deficits and cognitive impairment. ${ }^{14}$ Atrophy is also often observed and can be seen as dilation of the ventricles; thinning of the corpus callosum; and diffuse atrophy of the hippocampus, cerebellum, cerebrum, and brain stem. In approximately $30 \%-50 \%$ of cases, T2 hypointensities are found in the thalami and basal ganglia. These may be due to disruption of the physiologic axonal iron transport with secondary iron accumulation or to partition of toluene into the brain lipids. ${ }^{15,16,19,20}$

\section{Amphetamines and Derivatives}

\section{Cocaine}

Cocaine is a highly addictive drug due to its short biologic half-life and strong reinforcing properties which can lead to compulsive usage. ${ }^{21}$ The drug can produce local anesthesia by blocking nervous impulses; activate the parasympathetic nervous system by blocking the uptake of catecholamines and by blocking dopamine reuptake; and lead to a short intense euphoria with feelings of elation, increased energy, and alertness. Neurotoxic effects include agitation and seizures. In its most commonly used form (ie, cocaine hydrochloride), it is ingested via mucosal membranes. "Crack," the alkaloidal freebase of cocaine hydrochloride, can also be smoked. ${ }^{22}$

Most central nervous system complications induced by cocaine are vascular, with either hemorrhagic or ischemic strokes. Cocaine-related hemorrhages can be either intraparenchymal or subarachnoid and are twice as common as ischemic strokes. ${ }^{22,23}$ Approximately $40 \%-50 \%$ of patients have an underlying vascular pathology, such as a brain arteriovenous malformation or a brain aneurysm. Ruptures of the arteriovenous malformation or the aneurysm are most likely related to the elevated blood pressure and heart rate from the sympathomimetic effect of the drug (Fig 1)..$^{22,24,25}$ In cases without an underlying vascular pathology, the intraparenchymal hemorrhages are most often located in the basal ganglia and thalamus. Reperfusion hemorrhages from an ischemic stroke can also occur, and the risk of hemorrhage increases with the concomitant use of alcohol. ${ }^{26}$

While the pathomechanism of hemorrhagic stroke is straightforward, the causes for ischemic stroke are manifold, including cocaine-induced vasoconstriction, vasculitis (due to unknown additives/impurities), vasospasm, or the direct effects of cocaine on hemostasis. Cocaine enhances the responses of platelets to arachidonic acid, with increased levels of thromboxane, which leads to increased platelet aggregation resulting in concurrent myocardial and cerebral infarction from thrombosis. Cocaine also has procoagulant effects due to depletion of antithrombin III and protein C. ${ }^{27,28}$ Infarctions often involve the subcortical white matter within the MCA territory; however, they can be present anywhere in the brain. Most interesting, mesencephalic stroke seems to be more frequent when cocaine is used together with other amphet- 

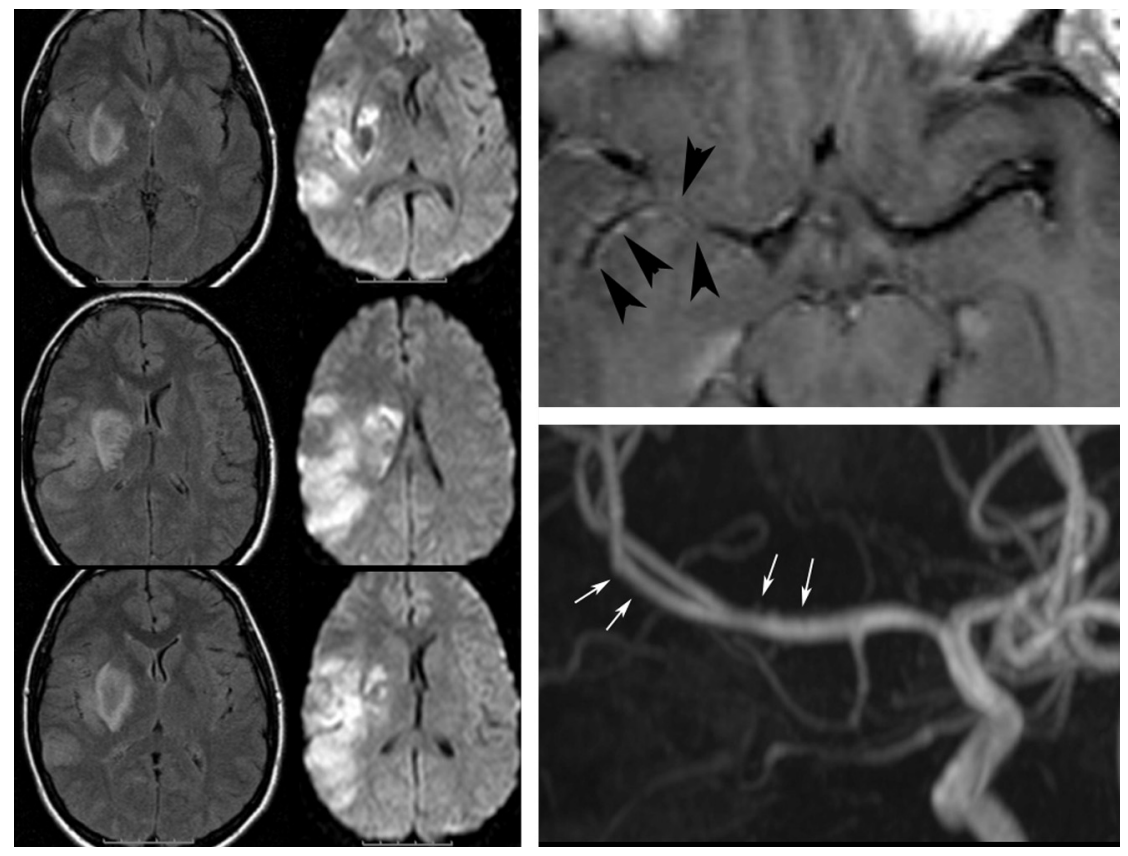

Fig 2. A 23-year-old woman woke up with a complete hemiplegia. Following a positive urine analysis, she admitted having taken considerable amounts of cocaine the night before. MR imaging demonstrates an MCA branch ischemia including involvement of the basal ganglia. T1-weighted postcontrast scans demonstrate abnormal vessel wall enhancement in the right MCA (arrowheads), and the time-offlight MRA shows some mild irregularities of the distal M1, potentially testifying to acute drug-induced vasculitis as the most likely cause for the ischemia (arrows).

amines. ${ }^{29}$ MRA may reveal focal narrowing of the main branches of the circulation with associated vessel wall contrast enhancement, which may favor local vasculitis as the causative mechanism (instead of vasospasm or vasoconstriction) (Fig 2 ). Cerebral atrophy is seen in chronic cocaine abusers in late stages. The frontal lobe is typically most severely affected, followed by the temporal lobes; chronic ischemia may be the underlying pathomechanism responsible for this atrophy. ${ }^{30}$

\section{Ecstasy}

Ecstasy or MDMA is a drug derived from methamphetamine, which is popular as a party drug because it has both stimulating and mild hallucinatory effects. In the acute phase, the drug users experience euphoria, increased selfconfidence, increased sensory perceptions, tachycardia, and hyperthermia. ${ }^{31,32}$ Trismus or acute psychoses may be present. The effect wears off after $24-48$ hours, with patients experiencing muscle aches, depression, fatigue, and decreased concentration.

MDMA causes acute rapid release of 5-HT, which is a potent vasoconstrictor from serotonergic synapses and leads to increased synaptic dopamine levels in several brain areas. ${ }^{33}$ Although its permanent neurotoxicity is still a matter of debate, there is evidence that stimulation of 5-HT2A receptors in small vessels will lead to prolonged vasospasm and result in necrosis of the involved regions of the brain. ${ }^{34}$ The occipital cortex and globus pallidus are the most vulnerable brain regions due to the high levels of 5-HT and 5-HT2A receptors. $^{35-37}$ Ischemia is, therefore, more likely to occur than hemorrhage and often involves small vessels (Fig 3). ${ }^{38}$

\section{Opioids and Derivatives}

Heroin is the most commonly abused drug within this group and has the most significant adverse effect on the brain. ${ }^{39}$ Other derivatives include morphine, hydrocodone, oxycodone, hydromorphine, codeine, and other narcotics such as fentanyl, meperidene, methadone, and opium. Three types of receptors are activated by heroin: Stimulation of the $\mu$ opioid receptor will result in analgesic effects, euphoria, respiratory depression, and miosis, while stimulation of the $\kappa$ and $\delta$ opioid receptors causes analgesic, dysphoric, and psychomimetic effects. ${ }^{40}$ Both acute and chronic effects of heroin on the brain have been described and include neurovascular disorders, leukoencephalopathy, and atrophy. ${ }^{39,41}$ In addition to these primary or direct effects of heroin on the brain, secondary complications have to be differentiated. These are related to added impurities (either lipophilic additives ["cutting"] or crystalline impurities), and they may also be secondary to associated diseases (such as infections or epilepsy).

Ischemia is the most commonly encountered acute neurovascular complication caused by heroin. The proposed pathomechanisms are manifold (similar to ischemia related to cocaine) and include the following: 1) direct effects of the heroin with reversible vasospasm from stimulation of the vascular smooth muscles by the $\mu$ opioid receptors, 2) vasculitis from immune-mediated responses (which may be related to the heroin itself or to impure additives), or 3 ) embolic events from crystalline impure additives (Fig 4). ${ }^{42,43}$ Ischemia is more often encountered following intravenous injection compared with other routes such as oral ingestion or inhalation. ${ }^{43}$ The globus pallidus is the most commonly involved location, and infarcts in this location are present in approximately 5\%-10\% of chronic heroin abusers. ${ }^{44}$ In addition to acute ischemia, white matter changes from microvascular disease can be seen in chronic heroin abusers. Diffuse, symmetric, and bilateral T2 hyperintensities may be perceived in the subcortical or periventricular white matter, compatible with vascular leukoencephalopathy. However, these changes are commonly not as severe as in chronic cocaine abusers.

An important complication is heroin-induced leukoencephalopathy, which is exclusively seen after heroin inhalation ("chasing the dragon") and is never seen following heroin in- 


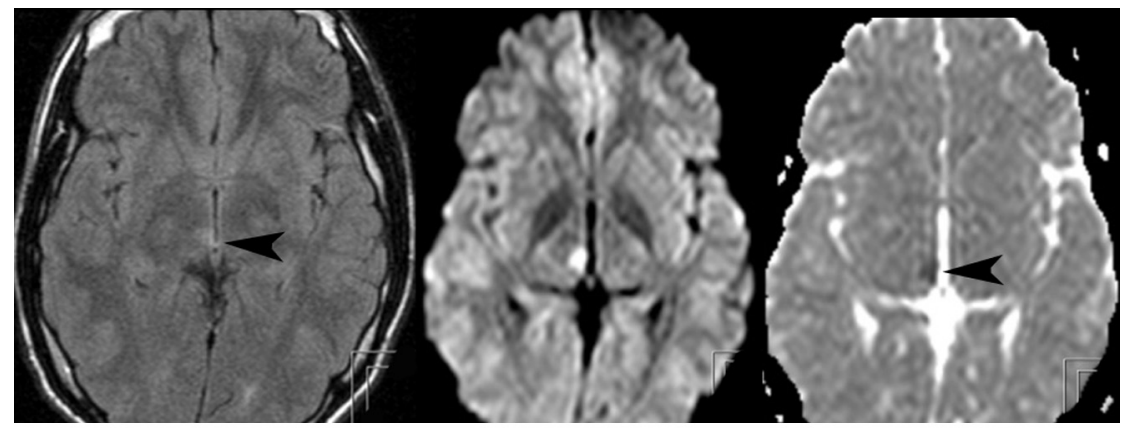

Fig 3. This young male patient with a subacute onset of hemisensory deficit following ingestion of an unidentified psychoactive designer drug has a small area of cytotoxic edema with fluid-attenuated inversion recovery hyperintensity and decreased apparent diffusion coefficient value in his right mediodorsal thalamus, which is classically supplied by thalamoperforators arising from the distal posterior communicating artery (arrowheads). The time-of-flight MRA at $3 \mathrm{~T}$ demonstrates a focal narrowing of this artery at the presumed origin of the thalamoperforators supplying the infarcted region (arrows). Drug-induced vasospasm or local vasculitis is presumed to be the cause for his acute ischemia.


Fig 4. Acute areas of ischemia in both the anterior and middle cerebral artery territories involving predominantly the gray matter as a result of a diffuse vasculitis with a "pearland-string" sign along the anterior cerebral artery, with an acute occlusion of the pericallosal artery (arrows) and lumina irregularities in multiple different vessels in a patient with intravenous heroine abuse. Whether the described vasculitic pattern is due to vasospasm (ie, the primary effect of the opioid), immune-mediated responses against the heroin or added impurities, or a primary infectious process of the vessel wall cannot be differentiated.
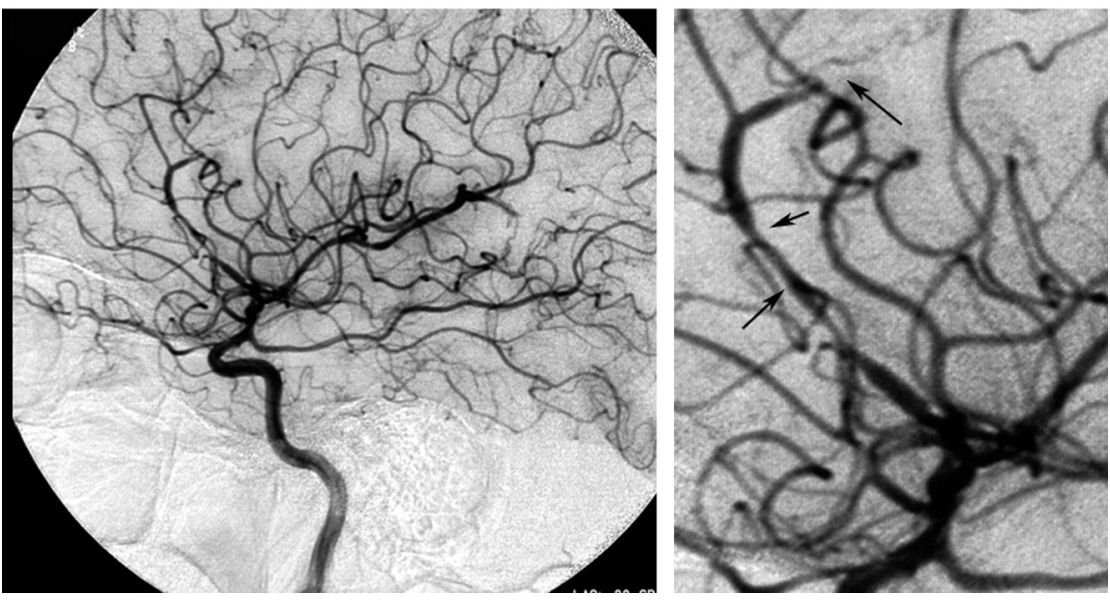

jection or oral ingestion. ${ }^{45-47}$ In heroin inhalation, the drug is heated over tinfoil and the emerging vapors are inhaled. Presumably due to activation of an as-of-yet-unknown substance, an acute leukoencephalopathy may occur with generalized brain edema (Fig 5). More common than this acute form, though, is the chronic subacute encephalopathy, which results in spongiform degeneration of the white matter, especially the corticospinal and solitary tracts, with associated multivacuolar degeneration of oligodendrocytes on histopathology (Figs 6 and 7)..$^{48,49}$
From a clinical point of view, the subacute heroin-induced leukoencephalopathy progresses from cerebellar symptoms to extrapyramidal symptoms, spasms, and hypotonic tetraparesis, eventually leading to the patient's death. The findings on MR imaging consist of T2 hyperintense lesions, predominantly in the white matter of the cerebellar hemispheres and posterior limb of the internal capsule (with sparing of the anterior limb), and the subcortical white matter, predominantly in the posterior aspect, only extending to the anterior aspect in severe cases or late stages. While in acute stages, diffusion re- 


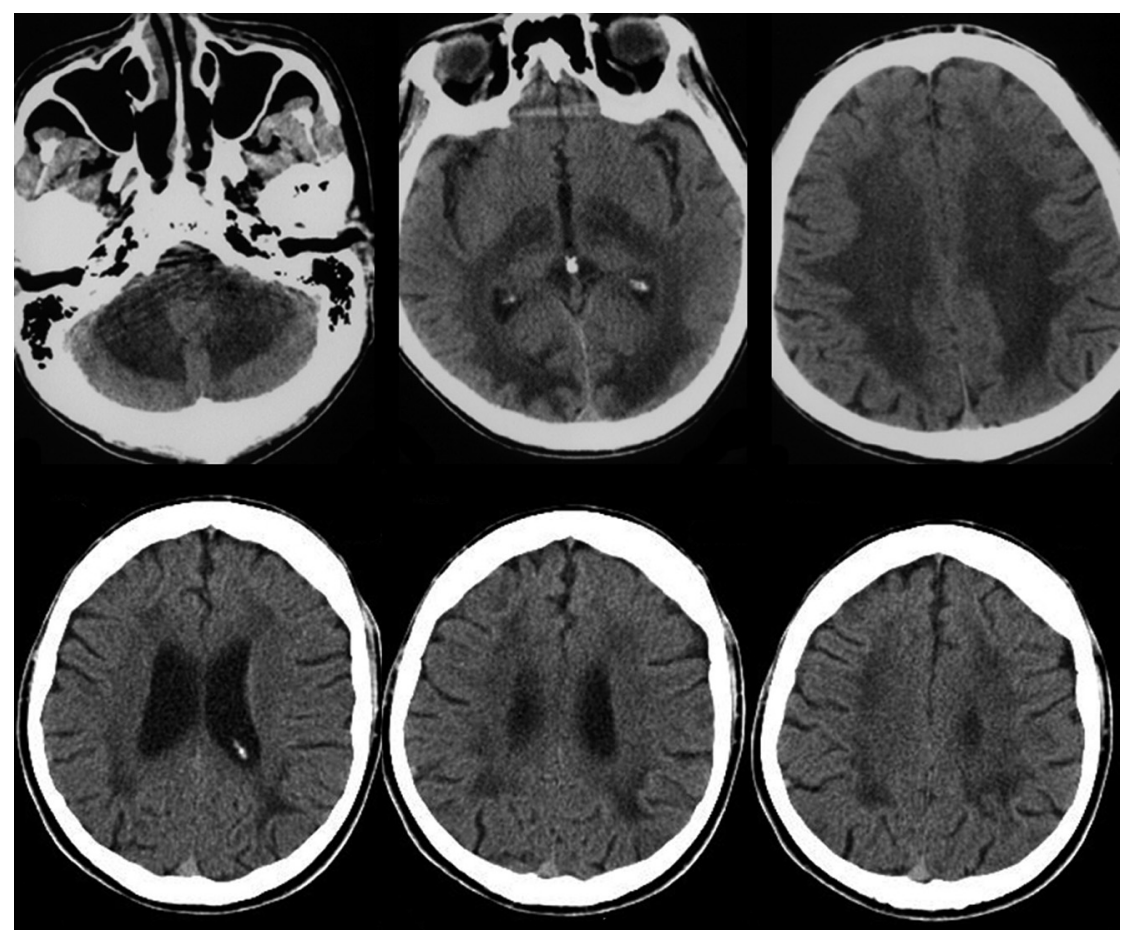

Fig 5. Acute (upper row) and chronic (after 6 months) effects following heroin inhalation (chasing the dragon). In the acute phase, a diffuse edematous swelling of the white matter is present both supra- and infratentorially. On follow-up after 6 months, the patient demonstrated mental retardation and persistent psychosis. CT demonstrates bilateral degeneration of the white matter with concomitant widening of the ventricles.
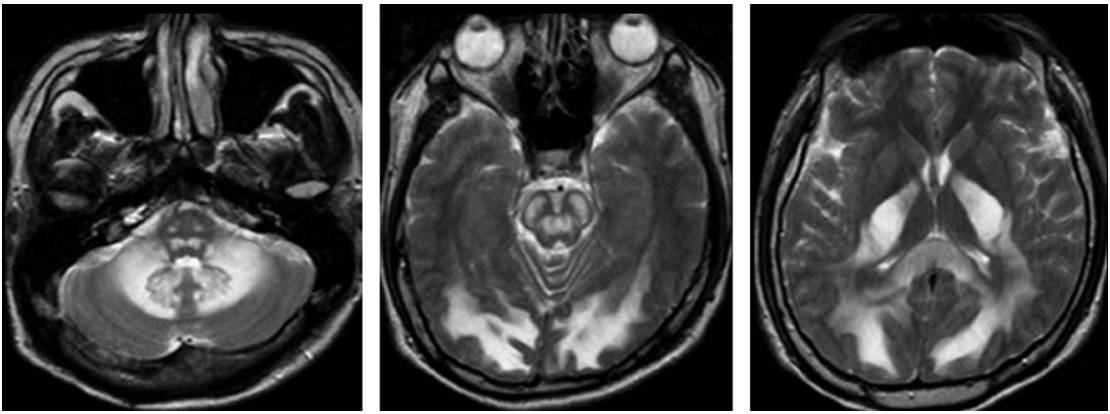

Fig 6. Chronic effects of heroin inhalation (typical form) Diffuse white matter changes are present, with sparing of the dentate nuclei and the cerebellar cortex and involvement of the corticospinal tracts and medial lemnisci. Clinically, this patient presented with a progressive spastic paraparesis.
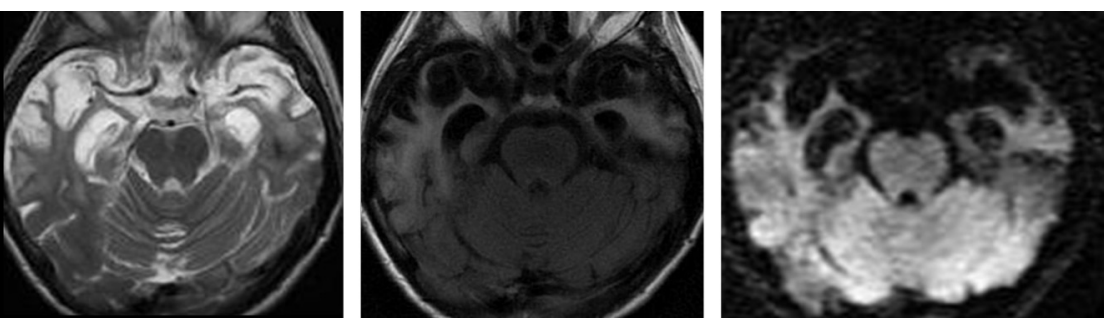

Fig 7. Chronic effects of heroin inhalation (atypical form This patient presented with psychosis and progressive dementia 20 years after she discontinued heroin inhalation (which she performed for nearly 10 years). Although not proved, the vacuolic degeneration speaks in favor of heroininduced leukoencephalopathy, which is predominant in the white matter of both temporal lobes.

striction is present, testifying to cytotoxic edema, the subacute stages will demonstrate an increased diffusion due to myelin breakdown.

Infections are the most important secondary complication of heroin abuse. CNS infections may occur following endocarditis due to nonsterile conditions associated with intravenous drug abuse. Up to $45 \%-58 \%$ of drug users with endocarditis will eventually develop neurologic complications, including septic emboli resulting in brain abscesses, and mycotic aneurysms caused by inflammatory destruction of the vessel wall. $^{50,51}$ The most common responsible organism is Staphylococcus aureus. ${ }^{52}$ Brain abscesses have the well-known and characteristic thin rim-like contrast enhancement surrounding a core that is hypointense on T1 and hyperintense on T2weighted scans and that demonstrates diffusion restric- tion. ${ }^{53,54}$ Extensive perifocal edema is present. Mycotic aneurysms can occasionally be seen on MRA; however, due to their small size and their peripheral location, they are better detected on conventional cerebral angiography and are seen as multiple small fusiform dilations involving the peripheral cortical arteries or perforators, with proximal and distal irregular narrowing and contrast stagnation in the capillary phase.

\section{Conclusions}

The main pathomechanisms related to the abuse of addictive drugs are ischemia, hemorrhage, and leukoencephalopathy related to excitotoxicity of the drug or its derivatives. While some imaging patterns are specific for a certain drug, others are overlapping. Clinical findings, especially in the acute setting, are often nonspecific, requiring the neuroradiologist to 
include drug-induced causes into the differential diagnoses of hemorrhage, ischemia, and diffuse white matter changes, especially in young patients.

\section{References}

1. Fowler JS, Volkow ND, Kassed CA, et al. Imaging the addicted human brain. Sci Pract Perspect 2007;3:4-16

2. Gatley SJ, Volkow ND. Addiction and imaging of the living human brain. Drug Alcohol Depend 1998;51:97-108

3. Lingford-Hughes AR, Davies SJ, McIver S, et al. Addiction. Br Med Bull 2003;65:209-22

4. Magalhaes AC. Functional magnetic resonance and spectroscopy in drug and substance abuse. Top Magn Reson Imaging 2005;16:247-51

5. Mena JC, Cuellar H, Vargas D, et al. PET and SPECT in drug and substance abuse. Top Magn Reson Imaging 2005;16:253-56

6. Moussouttas M. Cannabis use and cerebrovascular disease. Neurologist 2004; $10: 47-53$

7. Chang L, Chronicle EP. Functional imaging studies in cannabis users. Neuroscientist 2007;13:422-32

8. Quickfall J, Crockford D. Brain neuroimaging in cannabis use: a review. JNeuropsychiatry Clin Neurosci 2006;18:318-32

9. Iversen L. Cannabis and the brain. Brain 2003;126:1252-70

10. Mateo I, Pinedo A, Gomez-Beldarrain M, et al. Recurrent stroke associated with cannabis use. J Neurol Neurosurg Psychiatry 2005;76:435-37

11. Mathew RJ, Wilson WH, Humphreys DF, et al. Regional cerebral blood flow after marijuana smoking. J Cereb Blood Flow Metab 1992;12:750-58

12. O'Leary DS, Block RI, Koeppel JA, et al. Effects of smoking marijuana on brain perfusion and cognition. Neuropsychopharmacology 2002;26:802-16

13. Herning RI, Better WE, Tate K, et al. Marijuana abusers are at increased risk for stroke: preliminary evidence from cerebrovascular perfusion data. Ann NY Acad Sci 2001;939:413-15

14. Aydin K, Sencer S, Ogel K, et al. Single-voxel proton MR spectroscopy in toluene abuse. Magn Reson Imaging 2003;21:777-85

15. Yamanouchi N, Okada S, Kodama K, et al. White matter changes caused by chronic solvent abuse. AJNR Am J Neuroradiol 1995;16:1643-49

16. Aydin K, Sencer S, Demir T, et al. Cranial MR findings in chronic toluene abuse by inhalation. AJNR Am J Neuroradiol 2002;23:1173-79

17. King MD. Neurological sequelae of toluene abuse. Hum Toxicol 1982;1:281-87

18. Lazar RB, Ho SU, Melen O, et al. Multifocal central nervous system damage caused by toluene abuse. Neurology 1983;33:1337-40

19. Unger E, Alexander A, Fritz T, et al. Toluene abuse: physical basis for hypointensity of the basal ganglia on T2-weighted MR images. Radiology 1994;193: 473-76

20. Kamran S, Bakshi R. MRI in chronic toluene abuse: low signal in the cerebral cortex on T2-weighted images. Neuroradiology 1998;40:519-21

21. Williams MJ, Adinoff B. The role of acetylcholine in cocaine addiction. Neuropsychopharmacology 2008;33:1779-97

22. Brown E, Prager J, Lee HY, et al. CNS complications of cocaine abuse: prevalence, pathophysiology, and neuroradiology. AJR Am J Roentgenol 1992;159: $137-47$

23. Nolte KB, Gelman BB. Intracerebral hemorrhage associated with cocaine abuse. Arch Pathol Lab Med 1989;113:812-13

24. McEvoy AW, Kitchen ND, Thomas DG. Intracerebral haemorrhage and drug abuse in young adults. Br J Neurosurg 2000;14:449-54

25. Pozzi M, Roccatagliata D, Sterzi R. Drug abuse and intracranial hemorrhage. Neurol Sci 2008;29 (suppl 2):S269-270

26. Levine SR, Brust JC, Futrell N, et al. Cerebrovascular complications of the use of the "crack" form of alkaloidal cocaine. N Engl J Med 1990;323:699-704

27. Krendel DA, Ditter SM, Frankel MR, et al. Biopsy-proven cerebral vasculitis associated with cocaine abuse. Neurology 1990;40:1092-94
28. Nolte KB, Brass LM, Fletterick CF. Intracranial hemorrhage associated with cocaine abuse: a prospective autopsy study. Neurology 1996;46:1291-96

29. Kaufman MJ, Levin JM, Ross MH, et al. Cocaine-induced cerebral vasoconstriction detected in humans with magnetic resonance angiography. JAMA 1998;279:376-80

30. Rojas R, Riascos R, Vargas D, et al. Neuroimaging in drug and substance abuse. Part I. Cocaine, cannabis, and ecstasy. Top Magn Reson Imaging 2005;16: 231-38

31. Hurley RA, Reneman L, Taber KH. Ecstasy in the brain: a model for neuroimaging. J Neuropsychiatry Clin Neurosci 2002;14:125-29

32. Morgan MJ. Ecstasy (MDMA): a review of its possible persistent psychological effects. Psychopharmacology (Berl) 2000;152:230-48

33. Green AR, Mechan AO, Elliott JM, et al. The pharmacology and clinical pharmacology of 3,4-methylenedioxymethamphetamine (MDMA, "ecstasy"). Pharmacol Rev 2003;55:463-508

34. Reneman L, Habraken JB, Majoie CB, et al. MDMA ("Ecstasy") and its association with cerebrovascular accidents: preliminary findings. AJNR Am J Neuroradiol 2000;21:1001-07

35. Henry JA, Jeffreys KJ, Dawling S. Toxicity and deaths from 3,4-methylenedioxymethamphetamine (“ecstasy”). Lancet 1992;340:384-87

36. Spatt J, Glawar B, Mamoli B. A pure amnestic syndrome after MDMA (“ecstasy") ingestion. J Neurol Neurosurg Psychiatry 1997;62:418-19

37. Squier MV, Jalloh S, Hilton-Jones D, et al. Death after ecstasy ingestion: neuropathological findings. J Neurol Neurosurg Psychiatry 1995;58:756

38. Bae SC, Lyoo IK, Sung YH, et al. Increased white matter hyperintensities in male methamphetamine abusers. Drug Alcohol Depend 2006;81:83-88

39. Borne J, Riascos R, Cuellar $\mathrm{H}$, et al. Neuroimaging in drug and substance abuse. Part II. Opioids and solvents. Top Magn Reson Imaging 2005;16:239-45

40. Trescot AM, Datta S, Lee M, et al. Opioid pharmacology. Pain Physician 2008; 11(2 suppl):S133-153

41. Buttner A, Mall G, Penning R, et al. The neuropathology of heroin abuse. Forensic Sci Int 2000;113:435-42

42. Brust JC, Richter RW. Stroke associated with addiction to heroin. J Neurol Neurosurg Psychiatry 1976;39:194-99

43. Volkow ND, Valentine A, Kulkarni M. Radiological and neurological changes in the drug abuse patient: a study with MRI. J Neuroradiol 1988;15:288-93

44. Andersen SN, Skullerud K. Hypoxic/ischaemic brain damage, especially pallidal lesions, in heroin addicts. Forensic Sci Int 1999;102:51-59

45. Bartlett E, Mikulis DJ. Chasing "chasing the dragon" with MRI: leukoencephalopathy in drug abuse. Br J Radiol 2005;78:997-1004

46. Keogh CF, Andrews GT, Spacey SD, et al. Neuroimaging features of heroin inhalation toxicity: “chasing the dragon." AJR Am J Roentgenol 2003;180:847-50

47. Offiah C, Hall E. Heroin-induced leukoencephalopathy: characterization using MRI, diffusion-weighted imaging, and MR spectroscopy. Clin Radiol 2008;63:146-52

48. Schiffer D, Brignolio F, Giordana MT, et al. Spongiform encephalopathy in addicts inhaling pre-heated heroin. Clin Neuropathol 1985;4:174-80

49. Wolters EC, van Wijngaarden GK, Stam FC, et al. Leucoencephalopathy after inhaling "heroin" pyrolysate. Lancet 1982;2:1233-37

50. Pruitt AA, Rubin RH, Karchmer AW, et al. Neurologic complications of bacterial endocarditis. Medicine (Baltimore) 1978;57:329-43

51. Ramsey RG, Gunnar RM, Tobin JR Jr. Endocarditis in the drug addict. Am J Cardiol 1970;25:608-18

52. Tunkel AR, Pradhan SK. Central nervous system infections in injection drug users. Infect Dis Clin North Am 2002;16:589-605

53. Falcone S, Post MJ. Encephalitis, cerebritis, and brain abscess: pathophysiology and imaging findings. Neuroimaging Clin N Am 2000;10:333-53

54. Leuthardt EC, Wippold FJ 2nd, Oswood MC, et al. Diffusion-weighted MR imaging in the preoperative assessment of brain abscesses. Surg Neurol 2002; 58:395-402, discussion 402 